\title{
Biologically Inspired Trajectory Generation for Swarming UAVs Using Topological Distances
}

\author{
Gonzalo A. Garcia \\ Center for Remote Sensing for Ice Sheets \\ University of Kansas \\ Lawrence, Kansas \\ gagarcia@ku.edu
}

\author{
Shawn S. Keshmiri \\ Department of Aerospace Engineering \\ University of Kansas \\ Lawrence, Kansas \\ keshmiri@ku.edu
}

\begin{abstract}
This paper presents a trajectory generation logic for a swarm of unmanned aerial vehicles which uses topological distances instead of metric values. Biologically inspired, this method evades exploiting distance measurements in the absence of such capabilities, as it happens in swarming birds. This newly developed trajectory generation logic provides a stable swarming logic which resembles a large aggregation of birds. The hypothesis of this work is that without depth perception, swarming birds rely primarily on their perception of other birds to estimate proximity and adjust repulsive and attractive forces in real-time. From previous biological behavior studies, it is known that a homogeneous and orderly aggregation is exhibited by swarming birds locating themselves side by side rather than in front or behind each other. This behavior, which is based on the relative spatial proximity perception to their nearest neighbors in the flock, allows birds to converge into a highly cohesive and coordinated formation without explicit control of inter-agent distances. Inspired by this biological pattern, a trajectory generation logic is designed and tested in controlling a multiagent unmanned aerial systems without the use of interdistances. A six degrees of freedom nonlinear dynamic model for an unmanned aircraft is used for simulation purposes. Each vehicle is provided with a decentralized agent-based robust nonlinear model predictive controller and a set of nonlinear guidance laws for trajectory tracking. The formulation of the proposed trajectory generation, as well as the selected aircraft control, constitute a truly decentralized technique, that allows for an unlimited number of agents within the swarm, as no centralized computations are made.
\end{abstract}

Keywords-Nonlinear modeling predictive control; biologically inspired swarm trajectory generation.

\section{INTRODUCTION}

Biological groups, such as flocks of birds, are remarkably effective at maintaining optimized group structure, detecting and avoiding obstacles and predators, and performing other complex tasks. The paper is inspired by the interactions found in large swarms of birds, where remarkable patterns known as emergent behaviors are achieved by the following of simple rules. Such impressive inter-agent coordination is accomplished despite their natural physiological constraints. Although individual agents have limited sensing capability and cannot see the whole formation, they can form a flock with no apparent leader, which implies the lack of a centralized command. This highly coordinated collective behavior emerges from localized interactions among individuals within a flock or swarm of birds.

The work done in [1] is the first 3D measurement of the internal structure of flocking birds. This work found a formation pattern between the closest birds in terms of angular distribution. In [2], an accurate 3D swarm of a few thousand birds was reconstructed to confirm that their local interaction does not depend on metric distances, but rather on "topological distance." It was concluded that topological distance is based on the relative spatial locations of the closest birds, and not on exact knowledge of inter-distances. This showed that each bird interacts on average with a fixed number of nearest neighbors (statistically between six and seven). Birds beyond the nearest neighbors were isotropically distributed and their relative location had no statistical significance. Based on this, a direct conclusion was that the formation is cohesive, even in the case of time variant density.

Models intended to replicate swarm dynamics and geometry have mainly been constructed around the premise of a metric distance measurement capability. Article [3] developed a local navigation logic using three simple rules: (i) birds move in the same direction as their closest neighbors; (ii) birds remain close to their neighbors; and (iii) birds avoid collisions using inter-agent distances. Several models have been developed utilizing the same concepts trying to close the gap between the simulated models and actual behaviors.

Commonly, swarms of birds are modelled through the definition of adjacent zones of each bird, where the rules between pairs of individuals (as defined in [3]) are activated. The work in [4] proposes a fuzzy logic approach to determine each artificial animal (animat) dynamics. Within a concentric circular zone, a set of fuzzy rules translates the information of distance, angular offset, and relative differences in direction of flight speed into individual corrections. In [5] a probabilistic scenario is defined where the rules are executed based on an exponential distribution as a function of the inter-individual distances. Randomly pairs of birds are selected to be updated. Their results were able to replicate original field measurements in [2], supporting the idea of anisotropy within the nearest neighbors. The work in [6] proposes adaptive and time-varying zones to allow birds to interact with a fixed number of nearest neighbors. Their results resembled qualitatively the empirical 
data collected in [2]. The work in [7] proposes a different approach in the selection of the nearest neighbors. Voronoi cells are used to isolate the adjacent birds, and a Delaunay triangulation is then used to define the topologic interactions pairs between adjacent cells. The birds' actions are computed based on concentric zones, mainly based on inter-individual distances. A different approach, where no model is assumed a priori, is done in [8]. A minimally structured (maximum entropy) model obtains comparable results with respect to cited measurements, despite the fact that no causal-effect logic is incorporated.

Most birds do not have a wide depth perception. Depth is perceived through each eye providing a slightly different view of the same scene when there is an overlap in the field of vision. This is known as binocular vision. Although some birds have both eyes facing forward (such as owls), their binocular vision is not believed to be associated with the perception of distance, also called stereopsis [9]. Based on these conditions it seems unfeasible that birds in swarms adjust their positions based on distance values.

Our work proposes to base the individual corrective actions on a closeness and remoteness comparison logic between opposed surrounding birds with no distance measurement. It is based on a topological or qualitative distance comparison (as opposed to metric or quantitative, using the terminology of [2], and related references), reacting to keep themselves at the center of the surrounding birds. A feeling of "closer to" or "further away from" is used to correct the speed of advance of the swarm and to indirectly reduce the distance difference between spatially opposed birds. A homogeneous swarm speed realistic scenario. The aircraft are controlled in a decentralized way by a robust nonlinear model predictive controller (NMPC), taken from [10-11].

\section{SWARMING MODEL BASED ON TOPOLOGICAL DISTANCE}

\section{A. Biologically Inspired Trajectory Generation Concept}

Biologically inspired, the main premise of the proposed swarming model is the absence of inter-agent distance measurement, which is referred as metric distance (terminology used in [2]). The main hypothesis is that agents within a swarm, especially the interior ones, have no other alternative other than to keep themselves as much equidistant as possible from surrounding closest agents. This seems natural, particularly for swarms with large number of members where interior agents have their vision or other sensors in general hindered and could not take a different role (for example, as a temporary leader). As biological agents lack the ability to measure distances, it is reasonable to postulate that they have the ability to discern the relative closeness or remoteness to their neighbors and use this comparison to keep themselves in a centered position. The simplest version of this comparative approach would assume that all agents are from the same species and share similar properties, mainly size. This similarity would allow a sense of relative closeness by comparing their relative perceived size, but this capability by itself is not enough to keep the cohesion. A biologically preferred distance between agents and a preferred speed are assumed to be predefined and common for all members of the swarm.
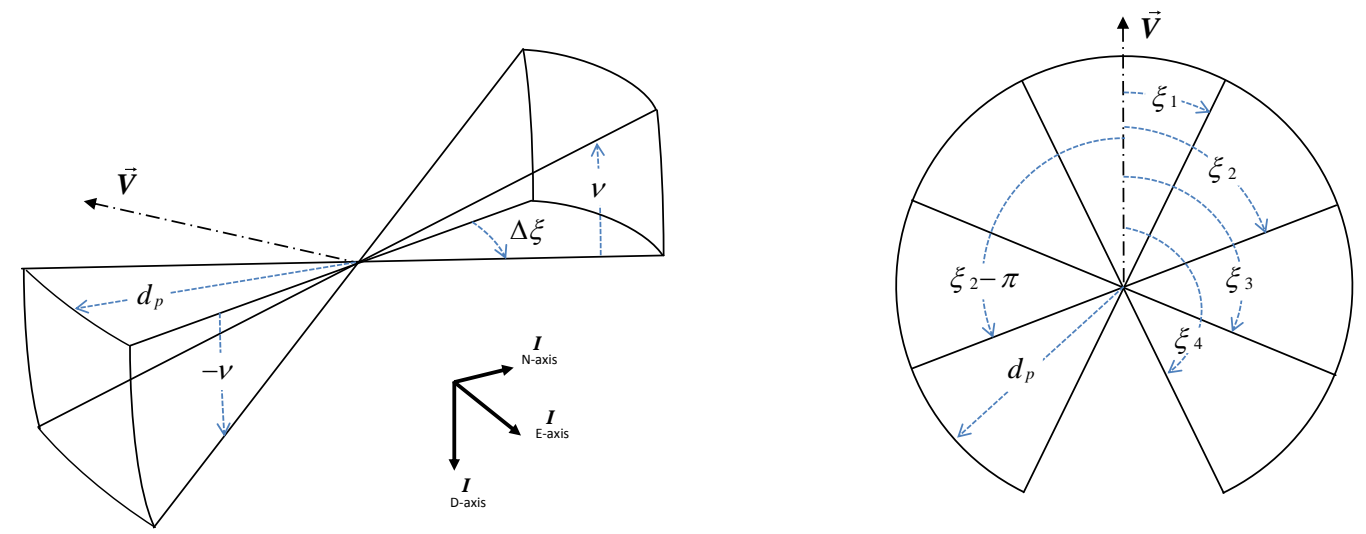

Figure 1. Sectored-based Model: 3D View (left) - 2D View (right).

and direction of motion, together with orderly aggregation, emerge as a consequence of this local and approximate spatial reasoning approach, without explicit control of these quantities.

With work in [2] as an empirical reference, biologically inspired and novel 3D guidance logic is been proposed, developed, and tested, with promising results in stability and robustness. Unmanned aerial systems (UASs) were chosen to test the logic, emulating the agents within the swarm, contributing with their nonlinear dynamics to allow a more
The space surrounding each agent is divided into solid angles (defined by a range of azimuth and elevation angles) and a radius. These ranges are referred to the agent's position and the direction of the speed vector $\vec{V}$, and horizontally leveled with respect to the inertial coordinate system $\boldsymbol{I}$. As shown in Figure 1, the space is divided into six lateral azimuth ranges, $\left\{\xi_{1}, \xi_{2}\right\},\left\{\xi_{2}, \xi_{3}\right\},\left\{\xi_{3}, \xi_{4}\right\},\left\{\xi_{4}-\pi, \xi_{3}-\pi\right\}$, $\left\{\xi_{3}-\pi, \xi_{2}-\pi\right\}$, and $\left\{\xi_{2}-\pi, \xi_{1}-\pi\right\}$, a forward looking sector $\left\{\xi_{4}-\pi, \xi_{1}\right\}$, and two vertical elevation angles, $v$ and $-v$, generating twelve lateral sectors and two forward sectors. Each 
of these spherical sectors are bound by the radius $d_{p}$. Motivated by bird's physiology, a blind sector is consider to point backwards. Relative closeness is evaluated between opposed sectors (for example, between sector $\left\{\xi_{2}, \xi_{3}\right\}, v, d_{p}$ and sector $\left.\left\{\xi_{3}-\pi, \xi_{2}-\pi\right\},-v, d_{p}\right)$. The number of sectors and their width have been selected arbitrarily but based on a natural biological constraint, assuming that birds cannot concurrently manage a large number of narrower sectors.

The hypothesis in this project, which is tested with unmanned vehicles, is that no cohesion, separation keeping, or alignment happens deliberately, but only an individual autocentering process. Based on the relative closeness perception, locally around each agent, a cohesive swarm congregation emerges globally. Possibly as a consequence, a fixed number of closest neighbors (6-7 agents as reported in [2]) distributed mainly to the sides, arises as a local pattern, and globally the density appears to become constant, independent of the swarm size. The majority of the models based on or derived from the three rules presented in [3], that allow for a cohesive aggregation of birds, are based on distances. The presented logic reproduces the same patterns achieved by those models without resorting to distance measurements, but to relative closeness perception.

In the present work, and as opposed to previous studies and modeling, no distance is used at the guidance level; although some works slightly departs from this restriction. For example, in [6] an adaptive interacting range is adapted online to interact with a predefined number of birds (based on averaged positions and speeds of the closest birds, with a simplified aerodynamic model), and its self-perception relative to the imaginary reference to the nearest neighbors. The research in [4] makes a step towards a more realistic approach by doubting that birds are able to perform sophisticated or time-intensive mathematical calculations. It shows that there must be simple logics to follow in the swarm generation, by questioning that birds are able to perceive the precise information on which most existing mathematical models are based. The research presented in this paper goes a step further than [4] by judging perceptions of distances and angular locations through a simple comparison of relative closeness/remoteness with surrounding birds. This is what probably a human being would do to avoid collisions if forced to run in a dense crowd, without much analysis. More complex logics are probably beneath swarm conformation, but their characterizations are not the objective of this research, and this work is not intended to fully characterize the empirical measurements; it only pursues to test the presented hypothesis with simulated aerial vehicles.

Three primary drives (attraction to flockmates, repulsion from flockmates and polarization with flockmates) are commonly used to achieve swarming; this logic only needs a single, namely, the bird keeps itself at the approximated center with the surrounding neighbors, while flying at a common direction and speed. A sensing range and a preferred distance in terms of relative sizes of neighbors are also defined and assumed common to all birds within a cluster.

\section{B. Trajectory Generation Description}

In this subsection a high level algorithm is outlined and tested with two different number of agents. The steps conform a decentralized guidance logic, run by each member separately. At each sample time the follow steps are executed:

- Determine the closest agent in each lateral sector defined in Figure 1, discard any other agent existing within the sectors.

- For each of the closest agents for lateral sectors, compare the relative size of all pairs of opposing sectors (for example, between sector $\left\{\xi_{2}, \xi_{3}\right\}, v, d_{p}$ and sector $\left\{\xi_{3}-\pi, \xi_{2}-\pi\right\},-v, d_{p}$, etc.), and determine the closest in each pair.

- Determine the closest agent in the forward sectors defined in Figure 1, discard any other agent.

- For each forward sectors, based on the size of the closest agent, determine its relative location with respect to the forward preferred distance (as seen below, these sectors are defined with a larger predefined distance or equivalently a smaller predefined size for the closest agent with respect to lateral sectors, taking into account the difference in relative speed between lateral agents and the forward ones).

- If no closest neighbor exists in one of the lateral sector pairs, apply the same logic as with the forward sectors, using the lateral preferred distance.

- Per each pair of opposed lateral sectors, both containing a closest neighbors, generate a speed correction in the direction of the sector's center of the furthest neighbor. If similar relative size is determine, no correction is made.

- Per each forward sector, create a correction in the opposed direction of flight if the closest agent is perceived closer to the preferred forward distance, and in the flight direction if it is perceived beyond the preferred forward distance.

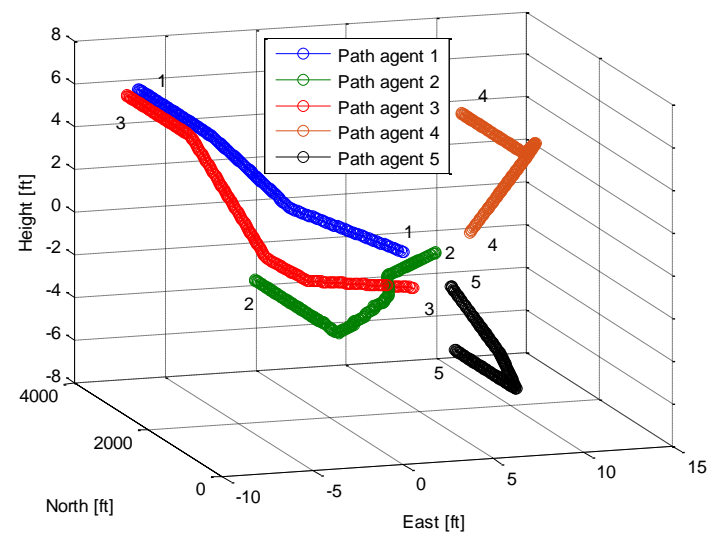

Figure 2. Agents Flight Path. 
- If there are no agents in any of the forward sectors and in both sectors any of the lateral pairs of sectors, generate no respective correction.

- Apply the vector summation of all corrections to the underlying swarm speed.

To examine the logic, two different settings are tested with different numbers of agents and initial locations. For both setups, the base speed is defined as 170 [ft/s] flying north.

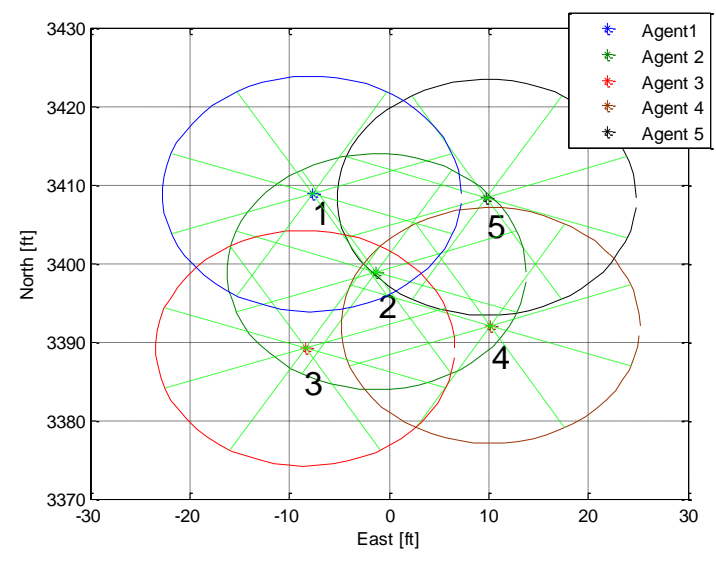

Figure 3. Final Horizontal Disposition.

The first testing considers 5 agents starting in a random position within 5 [ft] from each other, with a predefined size equivalent to a predefined lateral distance of 15 [ft], and a forward distance of 1.3 times the lateral one]. Individual corrections are $0.5[\mathrm{ft} / \mathrm{s}]$ per each pair of lateral sectors and forward sectors. Figure 2 shows the flight path, and Figure 3 shows the final location (only horizontally is shown for clarity) after 20 [s] of flight.

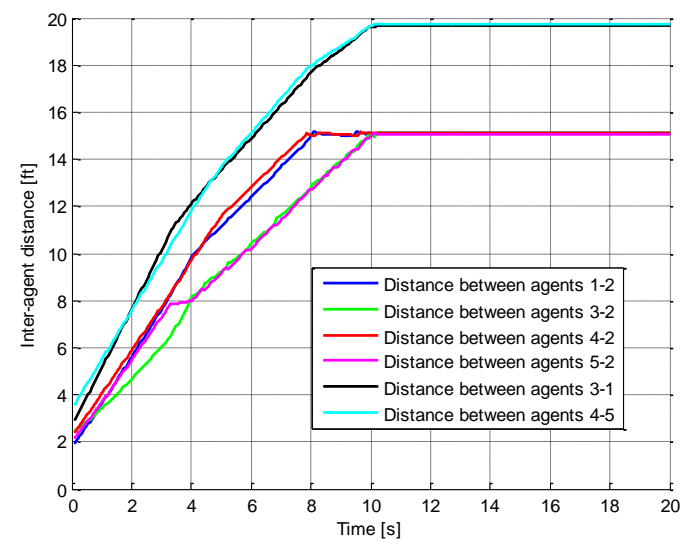

Figure 4. Inter-agent Distances.

Sectors are marked in Figure 3, where it is possible to see the inter-dependence between neighbor agents. In this particular run, all agents are individually linked only to agent 2. This is confirmed by the inter-agent distances, as shown in Figure 4; these pairs settled at 15 [ft]. Agents one and five are in the forward sectors of agents three and four, respectively. In this case, forward predefined size is expected to be smaller by a factor of 1.3, materialized by a larger forward distance of 19.5 [ft]. This allows the agents to keep more distance from other agents in front of them for safety reasons.

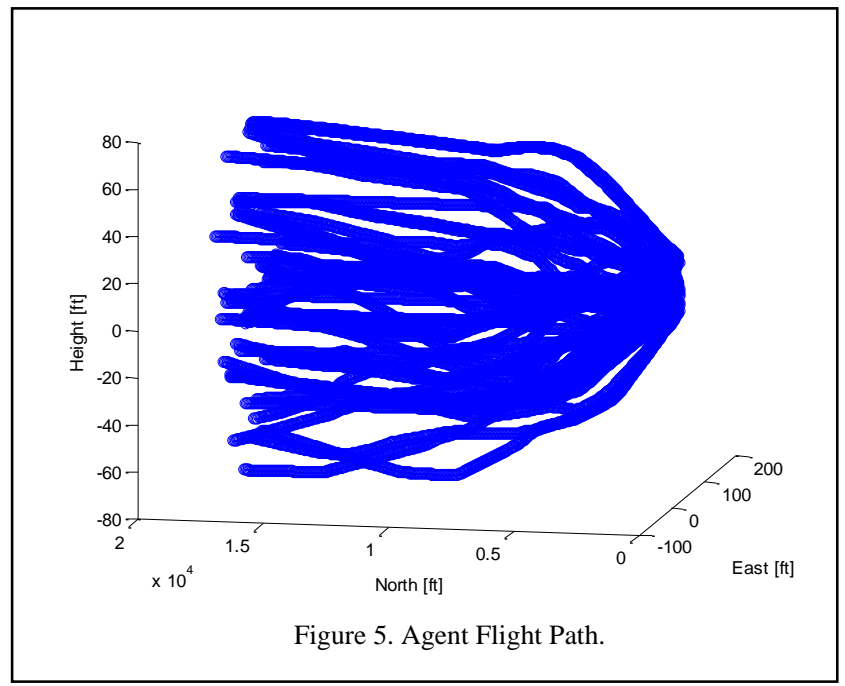

The second case considers 50 agents with predefined sizes equivalent to a 50 [ft] preferred lateral distance. Figure 5 shows their flight path, where after 100 [s] all agents expanded from a smaller region (as seen in Figure 6) to their final distances. This logic has proven to produce stable swarming.

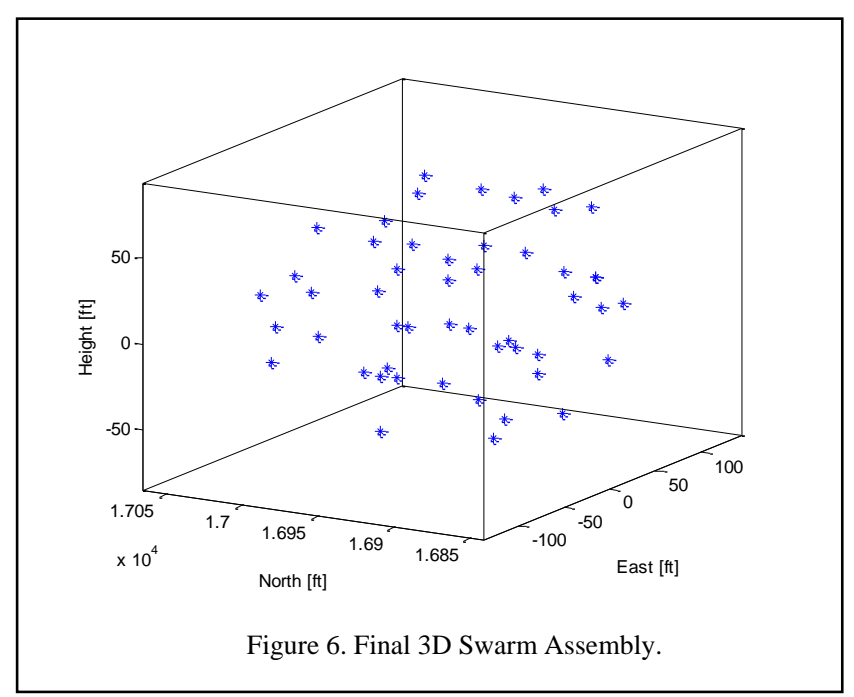

\section{SIMULATIONS USING UAV DYNAMICS}

In this section, aircraft dynamics plus guidance and control systems are used to test the swarming trajectories. Biologically inspired trajectory generation will be used to adaptively generate flyable trajectories to be followed by unmanned aerial vehicles (UAV). Each aircraft is equipped with an advanced nonlinear flight control system and a set of nonlinear guidance laws, for an accurate trajectory tracking, where both systems are acting in a decentralized way. The controller is based on robust nonlinear model predictive control, and the guidance law generates the controller commands based on the trajectory following errors, in an asymptotic way (see [11] for a detailed explanation). 
As detailed below, the guidance law aims to track the trajectory by following each new added point to the trajectory, as a result of the swarming algorithm. The guidance law basically generates the state commands, to be used by the predictive controller, necessary to asymptotically approach the moving point drawing the trajectory at each sample time.

\section{A. Aircraft Dynamics and Guidance Law}

Full aircraft dynamics are considered without any assumption or simplification. This modelling will be supplemented by a nonlinear guidance law (see [10]), allowing an arbitrary trajectory tracking.

Standardly, four coordinate systems centered in the aircraft's center of gravity are employed: coordinate system $\overline{\boldsymbol{I}}$, aligned with the fixed local flat earth geographical coordinate system $\boldsymbol{I}$; body coordinate system $\overline{\boldsymbol{B}}$, aligned with the structure of the aircraft; wind coordinate system $\overline{\boldsymbol{W}}$, aligned with the incoming airflow; and stability coordinate system $\overline{\boldsymbol{S}}$, rotated from $\overline{\boldsymbol{B}}$ by the trimmed airflow angles.

The model of a fixed wing aircraft is based on the sixdegrees-of-freedom rigid-body equation of motion derived from Newton-Euler equations, describing the combined translational and rotational dynamics, as shown:

$$
\begin{aligned}
& \dot{U}=R V-Q W-g \sin \theta+\left(X_{T}+X_{A}\right) / m \\
& \dot{V}=-R U+P W+g \sin \phi \cos \theta+Y_{A} / m \\
& \dot{W}=Q U-P V+g \cos \phi \cos \theta+Z_{A} / m \\
& \dot{P}\left(J_{x} J_{z}-J_{x z}^{2}\right)= \\
& \quad J_{x z}\left(J_{x}-J_{y}+J_{z}\right) P Q-\left(J_{z}\left(J_{z}-J_{y}\right)+J_{x z}^{2}\right) Q R+J_{z} L_{A}+J_{x z} N_{A} \\
& \dot{Q} J_{y}=\left(J_{z}-J_{x}\right) P R-J_{x z}\left(P^{2}-R^{2}\right)+M_{A} \\
& \dot{R}\left(J_{x} J_{z}-J_{x z}^{2}\right)= \\
& \quad\left(\left(J_{x}-J_{y}\right) J_{x}+J_{x z}^{2}\right) P Q-J_{x z}\left(J_{x}-J_{y}+J_{z}\right) Q R+J_{x z} L_{A}+J_{x} N_{A}
\end{aligned}
$$

These equation include the body velocities $\{U, V, W\}$, the angular rates $\{P, Q, R\}$, defined in $\mathrm{B}$, the attitude angles $\{\phi, \theta, \psi\}$ defined in $\overline{\boldsymbol{I}}$, and the aerodynamic forces $\left\{X_{A}, Y_{A}, Z_{A}\right\}$ and moments $\left\{L_{A}, M_{A}, N_{A}\right\}$ defined in $\bar{B}$. These aerodynamic quantities are modeled as functions of the aircraft states and throttle, elevator, aileron, and rudder servo deflections $\left\{\delta_{T}, \delta_{E}, \delta_{A}, \delta_{R}\right\}$ (see [11] for more detail about their modeling). Thrust force $X_{T}$, is only considered to act on the forward axis, and modeled as a linear function of $\delta_{T}$. Constants $\left\{J_{x}, J_{y}, J_{z}\right\}, J_{x z}, m$, and $g$, are the moments of inertia, product of inertia, mass, and gravity acceleration, respectively.

Servos deflections, defined in $\overline{\boldsymbol{B}}$, are driven by servo commands $\boldsymbol{u}=\left[\delta_{T}^{\text {cmd }}, \delta_{E}^{\text {cmd }}, \delta_{A}^{\text {cmd }}, \delta_{R}^{\text {cmd }}\right]^{T}$, and modeled as first order systems, with time constants $\tau_{T}, \tau_{E}, \tau_{A}, \tau_{R}$, respectively:

$$
\begin{aligned}
& \dot{\delta}_{T}=-\delta_{T} / \tau_{T}+\delta_{T}^{c m d} / \tau_{T} \\
& \dot{\delta}_{E}=-\delta_{E} / \tau_{E}+\delta_{E}^{c m d} / \tau_{E} \\
& \dot{\delta}_{A}=-\delta_{A} / \tau_{A}+\delta_{A}^{c m d} / \tau_{A} \\
& \dot{\delta}_{R}=-\delta_{R} / \tau_{R}+\delta_{R}^{c m d} / \tau_{R}
\end{aligned}
$$

Equations in (1) and (2) are complemented with the rigid body kinematic equations:

$$
\begin{aligned}
& \dot{\phi}=P+\tan \theta(Q \sin \phi+R \cos \phi) \\
& \dot{\theta}=Q \cos \phi-R \sin \phi \\
& \dot{\psi}=(Q \sin \phi+R \cos \phi) / \cos \theta
\end{aligned}
$$

and the local position equations:

$$
\begin{gathered}
\dot{p}_{N},=U \cos \theta \cos \psi+V(-\cos \phi \sin \psi+\sin \phi \sin \theta \cos \psi)+ \\
W(\sin \phi \sin \psi+\cos \phi \sin \theta \cos \psi) \\
\dot{p}_{E}=U \cos \theta \sin \psi+V(\cos \phi \cos \psi+\sin \phi \sin \theta \sin \psi)+ \\
W(-\sin \phi \cos \psi+\cos \phi \sin \theta \sin \psi) \\
\dot{p}_{H}=U \sin \theta-V \sin \phi \cos \theta-W \cos \phi \cos \theta
\end{gathered}
$$

with $\left\{p_{N}, p_{E}, p_{H}\right\}$ (North, East, Height), defined in $\boldsymbol{I}$.

The final aircraft model comprises equations (1) to (4), after replacing the body velocities with the equivalent dynamic equations for airspeed $V_{T}$ and airflow angles $\{\alpha, \beta\}$, defined in $\overline{\boldsymbol{W}}$ :

$$
\begin{aligned}
& \dot{V}_{T}=(U \dot{U}+V \dot{V}+W \dot{W}) / V_{T} \\
& \dot{\alpha}=(U \dot{W}-W \dot{U}) /(U U+W W) \\
& \dot{\beta}=\left(V_{T} \dot{V}-V \dot{V}_{T}\right) /\left(V_{T}^{2} \cos \beta\right)
\end{aligned}
$$

where $U=V_{T} \cos \alpha \cos \beta, V=V_{T} \sin \beta$, and $W=V_{T} \sin \alpha \cos \beta$. The final aircraft state vector is given by $\boldsymbol{x}=\left[V_{T}, \alpha, \beta, \phi, \theta, \psi, P, Q, R, \delta_{T}, \delta_{E}, \delta_{A}, \delta_{R}, p_{N}, p_{E}, p_{H}\right]^{T}$.

The aircraft model (1) to (5) is supplemented by the following attitude guidance equations:

$$
\begin{aligned}
& \phi_{c m d}=\tan ^{-1}\left\{k_{a_{\text {Lat }}} \cdot \eta_{\text {Lat }} \cdot\left(k_{p_{\text {Lat }}}+k_{\text {L }_{\text {Lat }}} / s\right)\right\} \\
& \theta_{c m d}=\tan ^{-1}\left\{k_{\text {aLon }} \cdot \eta_{\text {Lon }} \cdot\left(k_{\text {pLon }}+k_{\text {LLon }} / s\right)\right\}
\end{aligned}
$$

This addition allows an asymptotic tracking of the timevarying segment ( $\vec{a}[t]$ to $\vec{b}[t])$, subtended between consecutive moving points $\overrightarrow{\boldsymbol{o}}[t]$, as detailed [10] and shown in Figure 7 (up). The guidance logic calculates the angle $\eta[t]$ between UAS inertial velocity vector $\vec{V}[t]$ and position vector $\overrightarrow{\boldsymbol{L}}[t]$. This vector is subtended from the current UAS position $\vec{p}[t]$ to a point $\overrightarrow{\boldsymbol{r}}[t]$ located at a fixed distance $|\overrightarrow{\boldsymbol{r}}-\overrightarrow{\boldsymbol{d}}|=\overline{\boldsymbol{d}}_{d r}$ from the closest point $\overrightarrow{\boldsymbol{d}}$. Component angles $\eta_{\text {Lat }}$ and $\eta_{\text {Lon }}$ are calculated between lateral and longitudinal components $\vec{V}_{L a t}$ and $\overrightarrow{\boldsymbol{V}}_{L o n}$ of $\overrightarrow{\boldsymbol{V}}$, and lateral and longitudinal components $\overrightarrow{\boldsymbol{L}}_{\text {Lat }}$ and $\overrightarrow{\boldsymbol{L}}_{\text {Lat }}$ of $\overrightarrow{\boldsymbol{L}}$, respectively; $k_{p}$ and $k_{i}$ are the design gains, and $\quad k_{\text {LLat }}=2\left|\overrightarrow{\boldsymbol{V}}_{\text {Lat }}\right|^{2} /\left|\overrightarrow{\boldsymbol{L}}_{\text {Lat }}\right| \quad$ and $k_{\text {aLon }}=2\left|\overrightarrow{\boldsymbol{V}}_{\text {Lon }}\right|^{2} /\left|\overrightarrow{\boldsymbol{L}}_{\text {Lon }}\right|$. A speed command is designed to drive the aircraft asymptotically to moving point $\overrightarrow{\boldsymbol{o}}[\boldsymbol{t}]$, along the time-varying segment, see Figure 7 (down):

$$
V_{c m d}=\left|v^{o}\right|-k_{V o}^{1} \tan ^{-1}\left(\frac{1}{k_{V o}^{2}} \operatorname{sign}\left(d_{o b}-d_{d b}\right) \cdot d_{d o}\right)
$$

In the above equation, $\left|v^{o}\right|$ is the magnitude of the moving point speed, $k_{V_{o}}^{1}$ and $k_{V_{o}}^{2}$ are two positive constants that shape the magnitude and rate of the speed correction, and $d_{o b}, d_{d b}$, 
and $d_{d o}$ are the distances from $\overrightarrow{\boldsymbol{o}}$ to $\overrightarrow{\boldsymbol{b}}$, from $\overrightarrow{\boldsymbol{d}}$ to $\overrightarrow{\boldsymbol{b}}$, and from $\vec{d}$ to $\overrightarrow{\boldsymbol{o}}$, respectively.

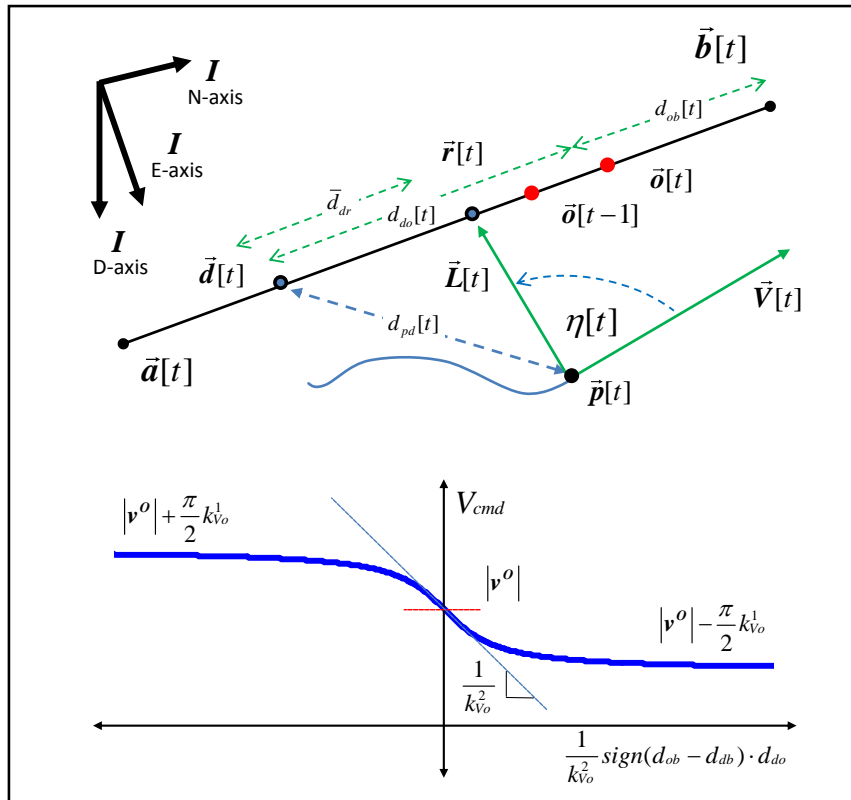

Figure 7. (up) Guidance Logic Geometry - (down) Speed Command.

Basically the guidance logic is divided in two parts. The one related to the angles $\eta_{\text {Lat }}$ and $\eta_{\text {Lon }}$ bringing the aircraft closer to the segment $\boldsymbol{a} \boldsymbol{b}$ between consecutive moving points, independently of the relative distance to the current moving point; and the one approaching the aircraft to the moving point $\overrightarrow{\boldsymbol{o}}$, regardless of the tracking of the subtended segment. The angles $\eta_{\text {Lat }}$ and $\eta_{\text {Lon }}$ are translated into pitch $\theta_{c m d}$ and roll $\phi_{c m d}$ commands through equation (6), and signed distance from $\overrightarrow{\boldsymbol{d}}$ to $\overrightarrow{\boldsymbol{o}}$ is translated to speed command $V_{\text {Tcmd }}$ with equation (7).

\section{B. Decentraliced Robust NMPC}

The control problem (based on [12]) for each aircraft independently, is formulated as the minimization of the $N-$ steps receding horizon cost function $V_{N M P C}=\sum_{k=1}^{N} \boldsymbol{e}_{k+1}^{T} \boldsymbol{Q} \boldsymbol{e}_{k+1}+\boldsymbol{u}_{k}^{T} \boldsymbol{R} \boldsymbol{u}_{k}$ for a known initial condition $\boldsymbol{e}_{1}$. This process is carried out in a decentralized way, with each aircraft running its own local NMPC algorithm including only its own variables. This approach allows the addition of unlimited number of new agents, without the risk of becoming numerically unfeasible, as could happens in centralized predictive control. The controlled and control sequences, $\left\{\boldsymbol{e}_{2}, \boldsymbol{e}_{3}, \cdots, \boldsymbol{e}_{N+1}\right\}$ and $\left\{\boldsymbol{u}_{1}, \boldsymbol{u}_{2}, \cdots, \boldsymbol{u}_{N}\right\}$, respectively, are obtained from the numerical minimization of the cost function $V_{N M P C}$, based on the aircraft model (1) through (5), as well as the guidance equations (6) and (7). The controlled output to be regulated to zero $\boldsymbol{e}=\left[e_{V_{T}}, e_{\theta}, e_{\phi}, e_{\beta}\right]^{T}$, include attitude tracking errors defined by $e_{\theta}=\theta_{c m d}-\theta$ and $e_{\phi}=\phi_{c m d}-\phi$, airspeed tracking error $e_{V_{T}}=V_{T c m d}-V_{T}$, and sideslip tracking error $e_{\beta}=\beta_{c m d}-\beta$ (in this work defined as $\beta_{c m d} \equiv 0$ ). Horizon time sample are denoted by the sub-index $k$. No explicit wind information is used in the prediction process as it is assumed as an unknown quantity. Wind can be injected during flight simulation as a disturbance, and the robustness extension of the NMPC should neutralize it, as explained below. Symmetric matrices $\boldsymbol{Q}$ and $\boldsymbol{R}$ are diagonal positive definite matrices.

Due to physical limitations in the actuators (i.e. maximum and minimum control surface deflections and maximum engine power supply), a set of input constraints was defined. Given the NMPC's numerical nature, not only aircraft modeling but also these maximum/minimum limits can be updated as required during flight. When these limits are reached during the numerical search, each violated constraint is adjoined to the cost function $V_{N M P C}$ by a time-varying Lagrange multiplier vector $\lambda$, creating a new cost function.

NMPC has no inherent robustness, being highly sensitive to modeling errors, and external disturbances. Two main streams of robustness augmentation are typically used. One approach is the min-max design, where the minimization of a cost function is performed jointly with the maximization of the effect of external disturbances. The linear analytical version of this method is the H-infinity control. The other technique is the shaping of characteristic closed loop functions by the addition of new dynamics. The first one, which is optimal by definition, quickly becomes unfeasible in real-time numerical applications such as NMPC, even for medium size order systems. The second approach, employed in linear designs as LQG or H-2, slightly departs from optimality, but does offer a tractable online solution. The present section integrates the technique to shape characteristic closed loop functions into the NMPC framework.

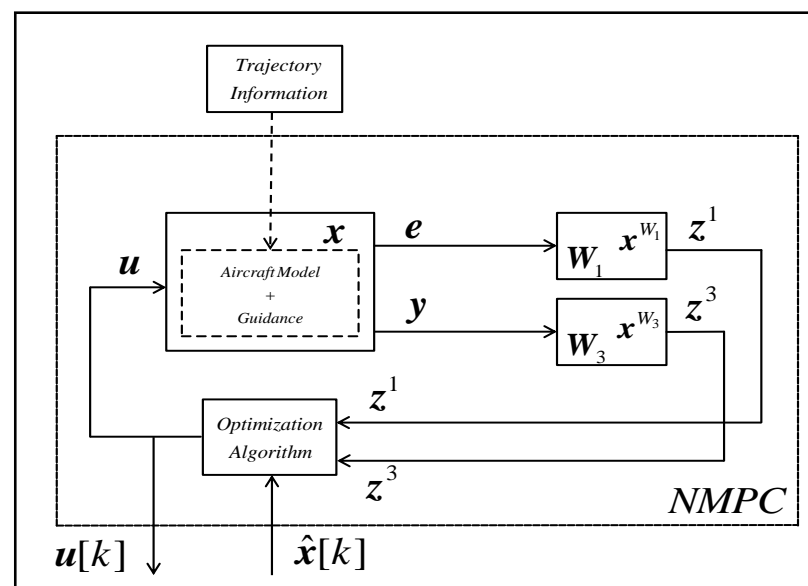

Figure 8. Robust NMPC (from [11]).

The robust NMPC developed in [12] was inspired by the robustness augmentation of the mixed sensitivity approach in the linear case. It is based on the assumption that reference signals, external disturbances, internal uncertainties, and measurement noises have bounded frequency content and are mutually exclusive. Disturbances and references are characterized by low frequency ranges, while measurement noises and model uncertainties are normally concentrated in a separated higher frequency range. This feature allowed the successful application of the mixed sensitivity concept in the NMPC framework, shaped by the frequency-dependent weighting matrices $\boldsymbol{W}_{1}$ and $\boldsymbol{W}_{3}$, added to the initial aircraft 
and guidance equations. This frequency modulation enabled the joint minimization of what were originally opposing closed loop objectives, specifically reducing the sensitivity function at lower frequencies $\left(\boldsymbol{W}_{1}\right)$ and concurrently reducing the complementary sensitivity function at higher frequencies $\left(\boldsymbol{W}_{3}\right)$. Given the assumption of the frequency ranges, the robust NMPC was able to reduce the effect disturbance, noise, and uncertainty, while following the references, without mutual interference.

The new minimizing objective, vector $z^{T}=\left[\left(z^{1}\right)^{T},\left(z^{3}\right)^{T}\right]$, is constructed from $\boldsymbol{e}$ and a new output defined by $\boldsymbol{y}=\boldsymbol{h}_{y}(\boldsymbol{x})=\left[V_{T}, \theta, \phi, \beta\right]^{T}$, as shown in Figure 8. The control problem is then reformulated as the minimization of $V_{\text {Robust NMPC }}=\sum_{k=1}^{N} \boldsymbol{z}_{k+1}{ }^{T} \boldsymbol{Q} \boldsymbol{z}_{k+1}+\boldsymbol{u}_{k}^{T} \boldsymbol{R} \boldsymbol{u}_{k}$ for a given initial condition $\boldsymbol{z}_{1}$, where sequences $\left\{\boldsymbol{z}_{2}, \boldsymbol{z}_{3}, \cdots, \boldsymbol{z}_{N+1}\right\}$ and $\left\{\boldsymbol{u}_{1}, \boldsymbol{u}_{2}, \cdots, \boldsymbol{u}_{N}\right\}$ are generated by the augmented aircraft model/guidance, derived from the addition of the weighting matrices $\boldsymbol{W}_{1}$ and $\boldsymbol{W}_{3}$.

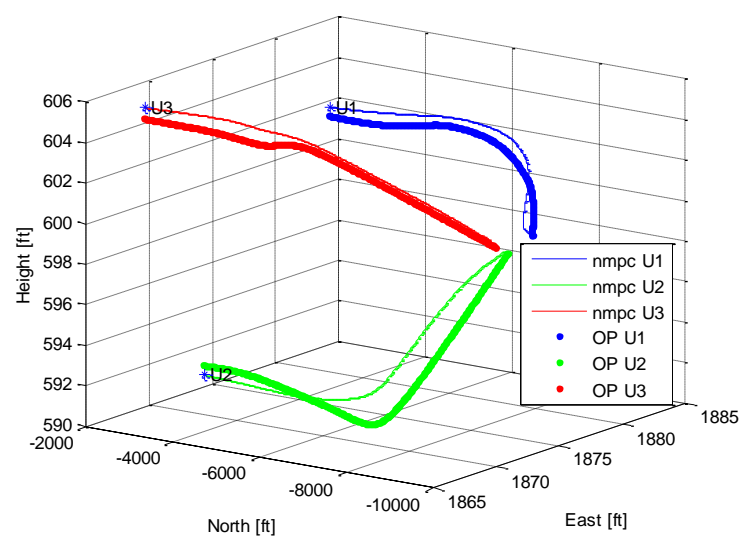

Figure 9. Trajectory and Aircraft Flight Path.

\section{Swarm Simulations}

The chosen aircraft for simulation is the UAS Meridian, flying north at 100 [knots]; see [12] for details of the aircraft. Three unmanned vehicles were simulated, starting at close proximity, at random position within 5 [ft] from each other, close to the point $-10,000$ [ft] North, 1,870 [ft] East, and 600 [ft] Height.

Figure 9 shows the trajectory and flight path of the aircraft (flying from right to left in the figure). The thick line represents the curve delivered by the trajectory generation logic based on

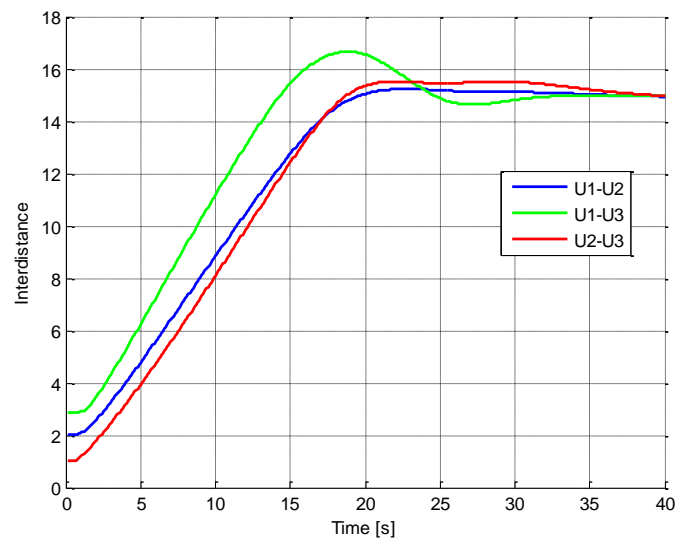

topological distances. As they fly North, they adjust their flocking behavior by following the trajectory with an equivalent preferred distance of 15 meters. Aircraft flight paths, are indicated by the thin line.

Figure 10 shows that after 40 seconds of flight, the intervehicle distance had approximately converged to the expected value of 15 feet. It is important to notice that the final location within the flock is not predictable, and could vary from flight to flight, as external disturbances could change.

Figures 11 and 12 show the 3D and horizontal zooming to the final location, in which aircraft has reached stability in flocking.

The simulation was designed with $N=20$ samples for the NMPC prediction horizon, and a sampling interval of $\Delta T=1 / 20$ seconds, consisting of a predicted flight time horizon of 1 second. Weighting matrices were constructed with transfer function $1 / s$ in $\boldsymbol{W}_{1}$ diagonal entries, and $(8 s+0.1) /(s+0.8)$ in $\boldsymbol{W}_{3}$. Attitude guidance parameters were chosen as $k_{\text {pLat }}=20 / 64, k_{\text {pLon }}=2.3 / 64, k_{\text {LLat }}=0$,

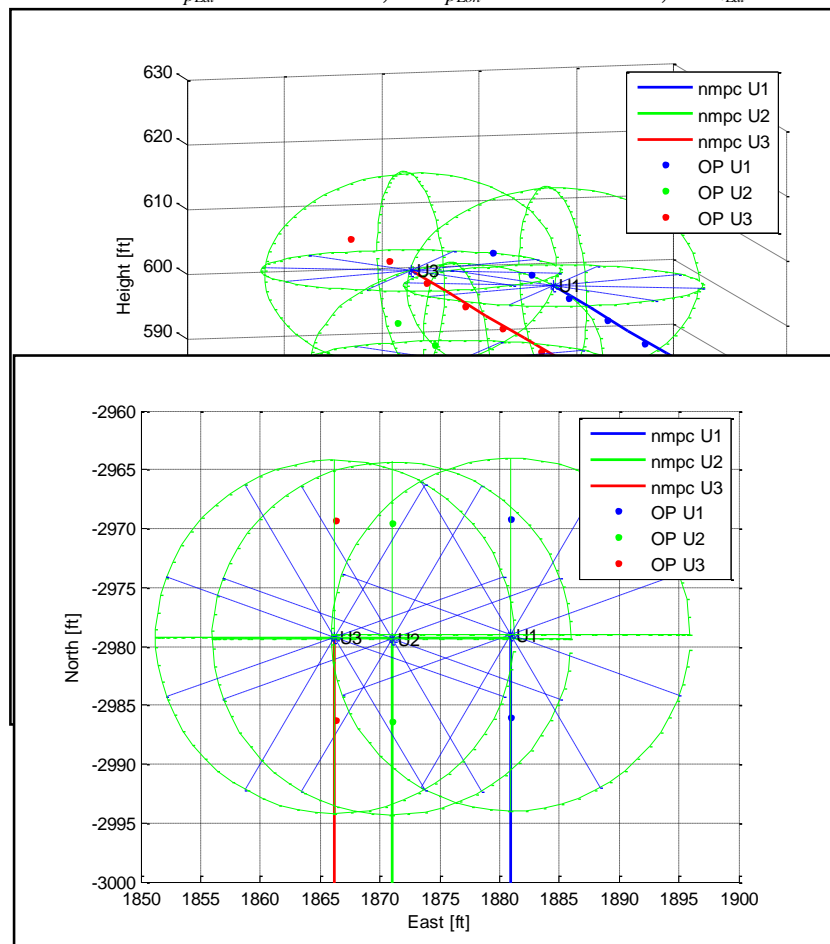

Figure 12. Final Aircraft Location (horizontal view).

$k_{i L o n}=0.2 / 64, \bar{d}_{d r}=1,400[f t], k_{V o}^{1}=1 / 5 \pi$ and $k_{V o}^{2}=100$.

\section{DISCUSSION}

Despite the acceptable results, the authors consider the trajectory generation logic to be preliminary. There are still several functions that have to be incorporated to make the process more robust. Simple but effective functionalities, preferably based on biologically inspired evidence, will be designed and tested to expand the guidance applicability. These include: 
- If an agent/aircraft belonging to a swarm has empty sectors, meaning that it is away from the swarm, it should use its sensor range capability (assumed to be larger than its preferred separation distance) to locate the swarm as a body, without any major detail, and get as close as possible. When a swarm is nearby, the aircraft will start using this topological distance logic.

- A qualitative distinction should be imposed between inner and boundary agent/aircraft. The logic now treats all agents equally, without any specific role assigned to any particular member. Biologically inspired logic can be advanced to further specialize the role of the boundary birds. It is the author's opinion that these birds probably have the capability to modify swarm shape and direction of movement, an attribute clearly forbidden for inner agents. This is because they have direct sensory access to external features (such as obstacles or potential depredators), as opposed to inner elements, which concentrate only on keeping themselves in the center of surrounding birds and avoiding collisions.

- A visual processing mechanism should be implemented with the presented idea to extract and compare relative sizes of surrounding aircraft during flight, as well as discriminating where the closest neighbors are and discarding agents that are further away. This is not a trivial task, but existing visual techniques could be used to expedite the design.

\section{CONCLUSIONS}

New trajectory generation logic has been developed inspired by the visible behavior of birds within a tight swarm. Based on perception of closeness and the relative location of neighboring agents, what has been called in the literature the topological distance, and with the absence of metric or exact values, the logic is able to provide a stable congregation of birds.

This behavior has been detected when birds are flying in very close proximity. They are mainly located side by side rarely having birds in front or behind at close distance, resulting in an orderly swarm that performs highly coherent maneuvers.

To test the idea, a trajectory generation logic was designed and implemented to command each aircraft in a multi-agent unmanned aerial system. Six degrees of freedom nonlinear dynamic models were used for simulation.

Using relative spatial closeness to their nearest neighbors in the flock without explicit knowledge of inter-agent distances, aircraft maneuvered to keep a coherent formation. For the inner loop a robust nonlinear predictive control approach was selected that accurately followed the outer loop commands. Both trajectory generation, and guidance and control logics are fully decentralized, without any explicit coordination or centralized approach within the formation, allowing an unlimited number of agents within the swarm.

Based on the simulation results, authors believe that the main assumption of the paper has been successfully tested, where without exact knowledge of the distance of surrounding neighbors, an relying primarily on their relative proximity perception, each aircraft effectively adjusted its repulsive and attractive forces in real-time, to keep a stable swarm.

\section{ACKNOWLEDGMENT}

This work was completed with funding from the Paul G. Allen Foundation (PGAFF) grant KUEA\#40956 and the National Science Foundation (NSF) Center for Remote Sensing of Ice Sheet (CReSIS) grant ANT-0424589, at the University of Kansas.

\section{REFERENCES}

[1] P. F. Major, and L. M. Dill, "The Three-Dimensional Structure of Airborne Bird Flocks", Behavioral Ecology and Socialbiology, vol. 4, 1978, pp. 111-122.

[2] M. Ballerini et al "Interaction Ruling Animal Collective Behavior Depends on Topological Rather Than Metric Distance: Evidence from a Field Study", PNAS, vol. 105, 2008, pp. 1232-1237.

[3] C. Reynolds, "Flocks, Herds, and Schools: A Distributed Behavioral Model", Computer Graphics, vol. 21, No. 4, 1987, pp. 25-34.

[4] I. Lebar Bajec, N. Zimic, and M. Mraz, "Simulating Flocks on the Wing: The Fuzzy Approach", Journal of Theoretical Biology, vol. 223, No. 2, 2005, pp. 199-220.

[5] N. W. F. Bode, D. W. Franks, and A. J. Wood, "Limited Interactions in Flocks: Relating Model Simulations to Empirical Data", Journal of the Royal Society Interface, vol. 8, 2011, pp. 301-304.

[6] C. Hemelrijk, and H. Hildenbrandt, "Some Causes of the Variable Shape of Flocks of Birds", PLoS ONE, vol. 6, e22479, 2011.

[7] A. Koplas, M. Busch, H. Li, I. D. Couzin, L. Petzold, and J. Moehlis, "How the Spatial Position of Individuals Affect Their Influence on Swarms: A numerical Comparison of Two Popular Swarm Dynamics Models", PLOS ONE, vol. 8, No. 3, e58525, 2013.

[8] W. Bialek, A. Cavagna, I. Giardina, T. Mora, E. Silvestri, M. Viale, and A. M. Walczak, "Statistical Mechanics for Natural Flocks of Birds", PNAS, vol. 109, no. 13, 2012, pp. 4786-4791.

[9] G. R. Martin, "What is Binocular Vision For? A Birds' Eye View", Journal of Vision, vol. 9, no. 11, art. 14, 2009, pp. 1-19.

[10] G. Garcia, and S. Keshmiri, "Online Artificial Neural Network Modelbased Nonlinear Model Predictive Controller for the Meridian UAS", International Journal of Robust and Nonlinear Control, vol. 23, 2013, pp. 1657-1681.

[11] J. Roskam, Airplane Flight Dynamics and Automatic Flight Controls (Part I), DARcorporation, Lawrence, Kansas, USA, 2003.

[12] G. Garcia, S. Keshmiri, and T. J. Stastny, "Robust and Adaptive Nonlinear Model Predictive Controller for Unsteady and Highly Nonlinear Unmanned Aircraft", IEEE Transactions on Control Systems Technology, Vol. 23, 4, 2014, pp. 1620-1627. 